\title{
Harnessing Green Cloud Computing- An Energy Efficient Methodology for Business Agility and Environmental Sustainability
}

\author{
Bharani Dharan $\mathbf{G}^{\mathbf{1}}$, Dr.Jayalakshmi $\mathrm{S}^{\mathbf{2}}$ \\ ${ }^{1} \mathrm{PhD}$. Research Scholar, Dept. of Computer Science, VISTAS, Pallavaram, Chennai-117, Tamilnadu, India. \\ mailto.bharanidharang@ rediffmail.com \\ ${ }^{2}$ Professor, Dept. Of Computer Applications, VISTAS, Pallavaram, Chennai-117, Tamilnadu, India
}

\section{ABSTRACT}

The role of Information, Communication and Technology (ICT) is important now-a-days and its impact on the nature is huge which emits more heat and $\mathrm{CO} 2$. Cloud Computing is playing an impressive role to face the challenges in IT Modern Digital Era to process the growing large volume of data and storing that data in Data Centers (DC) and provides the resources and applications to the customers with more scalability, flexibility, cost-effective, pay-on-demand basis and anything as-a-service in cloud but most of the prevailing cloud datacenter architectures are not aligned to green standards. To minimize the impact on cloud computing environment energy-efficient methods are required. Green Cloud is still under research and at nascent stage in its growth and implementation. Green cloud computing is an eco-friendly methodology helps to make the cloud computing environment with more power efficient, maximum resource utilization not by over-provisioning, less carbon footprints, minimized Green House Gas (GHG) emissions and reduction in E-wastages. In order to save cost, large enterprises come up with cutting edge technologies like Green Cloud Computing (GCC) by bringing them into the cloud environment to reduce the operational expenditures (OPEX) and allow the organizations to make less use of physical components and resources with virtualization techniques that requires only less cooling, less water consumption and minimized power consumption to keep the nature calm. The Cloud Service Providers (CSP) should upgrade their cloud DC infrastructure by eliminating the negative impacts which causes harm to the nature. Green Cloud Computing focuses on bringing the necessary characteristics and approaches in cloud environment like containerization, Designing Green Data center facility infrastructure, VM placement, Server Consolidation, Thin Clients, Green metrics, Green Network and Protocols, Green Data Management, Sustainable software development and safe disposable methods with $3 \mathrm{R}$ 's (Reuse, Reduce and Recycle). In this paper the discussions are about various elements and green cloud methodologies which are most needed to bring positive impact in our environment and to reduce metered bill for business profit margin and agility.

Key words : Cloud computing, Green Cloud Computing, Virtualization, Containerization, VM placement, Safe Recycle, Thin Clients, carbon footprints.

\section{INTRODUCTION}

With the tremendous growth of large enterprises comprises of datacenters with high speed networks increases the usage of more transactional applications like ERP (Enterprise Resource Planning), SAP (System Application Products in data processing), big data and millions of web search a day. These on-demand applications is handled by large Datacenters (DC) which contains some thousands of servers, storage, networks with cooling systems in its infrastructure [1]. These applications, platform, infrastructure has been provided by cloud computing as-a-service through pay-as-you-go model [2]. With cloud based approach, one can get the service through on-demand service request and the organizations do not need to invest more on Capital Expenditures (CAPEX) to acquire new hardware to run their business and the resource maintenance is completely hand-over to the third party based Cloud Service Providers (CSP) like Amazon, Google, Alibaba, which are operating giant data centers in major cities across the world. In the cloud computing user can store, manage and share their data without any hassle. On taking the advantage of cloud, medium or large level enterprises can save cost and time by configuring, managing, administering their on-premise data center is also very easy because whenever the updates or patches are released by the CSP it will be applicable for the clients without paying no more extra cost. So that an organization can concentrate on their core business activities to establish for competing by harnessing number of benefits in cloud such as faster development, accessing on-demand computing resources on cloud, test their products at very low cost. Cloud computing also offers large data storage for storing transactional or big data and data analytics to manage the huge volume of data with disk backup facilities with more 
fault tolerance and High Availability (HA) of data to the clients $24 \times 7$.

Cloud will provide a great market opportunity with on-demand services for most of the companies. IDC (International Data Center) has predicted the companies like CISCO, HP, IBM and the web-based companies like salesforce.com has invested large capital in establishing cloud Data centers (DC).

In cloud data centers the resources are virtualized and provided as-a-service on metered cost basis. Data centers require more power to operate the resources. For an example a typical data center with 2000 racks requires 20 Megawatt of energy to operate the resources with high Operational Cost (OPEX) [3]. In 2018 Gartner has estimated that Information and Communication Technologies (ICT) generate about $11 \%$ of $\mathrm{CO} 2 \neg$ emissions. Therefore, it is necessary in the upcoming years to keep decreasing the temperature in its limit. Thus the carbon emission and energy consumption by means of cloud DC has become a serious environment concern. Cloud Computing can provide a lot of benefits to the enterprises and its clients. However, the significant increase in cloud computing with Internet traffic and large growth of database volumes which could decrease the savings in energy. The below figure 1 depicts that the heat emits from cloud data centers and power grid affects the environment by high power usage and thereby decrease in revenue. Here in this cloud architecture broker does not maintain any metrics to maintain carbon emission levels, Power Usage Efficiency (PUE) which may lead to declination in Quality of Service (QOS).

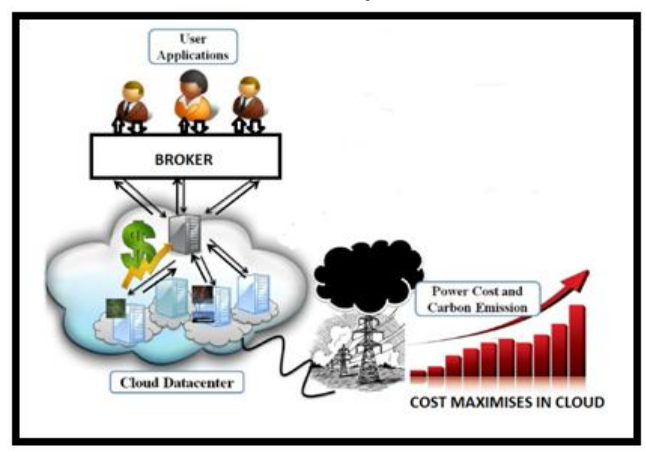

Figure 1: Cloud DC Architecture that consumes High Energy, Increased Cost and $\mathrm{CO}_{2}$

In the next few years there is a collective demand for the computing resources is expected to be high. So there is a need to build modern data center with greener technologies will only mitigate the higher utilization rates which will reduce energy and $\mathrm{CO} 2$ emissions. The contributors such as Environmental Protection Agency (EPA) of United States of America, Green peace have taken more initiatives to protect the environment by means of using eco-friendly efficient resources. To support with efficient methodologies in cloud computing environment, Green Cloud Computing (GCC) framework comprises of green broker with eco-friendly techniques will help to make the environment in a sustainable way.

\section{LITERATURE SURVEY}

Mostafa Sami et. al., [4] focused on server consolidation to limit the number of physical host or machines and it would be switched over to sleep mode until the task assigned to the virtual machine. K.Ganapathy et. al., [5] discussed about reduction of energy usage in cloud computing through decision based algorithm. A proposed method contains virtual machine in physical machine with job organizer. This method is based on Distributed service mechanism.

Fatima shakeel et. al., [6] explored that data center contains more number of hardware's with chipset which generates more heat and to control this problem, virtualization technique has been introduced to virtualize the servers and other resources in the Data Centers (DC) to reduce the energy by $35 \%$ on dividing a physical server to run different virtual machines at a time. On comparing the Dynamic Voltage Frequency Scaling (DVFS) algorithm, decentralized algorithm for resource allocation reduce the energy by $66 \%$. Fumiko Satoh et. al., [7] also concentrated on reducing the power consumption in data centers. They developed energy management system for cloud with Sensor Management Function (SMF) through advanced VM (Virtual Machine) allocation tool for future energy management. This mechanism helps to reduce $28 \%$ of energy in multiple DC. This function also helps to reduce carbon emissions.

Truong Duy et. al., [8] focused to reduce the energy in cloud through green scheduling algorithm with Artificial Neural Network Predictor (ANNP). This algorithm makes the server to predict the peak load from ' $t$ ' to the time take to restart. The number of server state is decided by peak workload. Rasoul Beik et. al., [9] proposes by adding energy aware layer in software model or architecture to calculate energy consumption in DC for energy efficiency.

Yamini et. al., [10] introduced the techniques and approaches like power management, virtualization, material recycling in green cloud was beautifully expressed. Nimje et. al., [11] introduced virtualization to attain security in cloud data centers for making it Green further. Buyya et. al., [12] contributed on green cloud architecture which contains carbon emission and green offer directories. These directories provide Green services. Green offer directory gives the information to the Green Broker about less $\mathrm{CO}_{2}$ emission to schedule the services.

Amusya Krishna Prity et. al., [13] has proposed an Ad-Hoc demand algorithm for improving the network efficiency in cloud data center. Beloglazov et. al., [14] proposed techniques with the offline and online deterministic algorithm with optimal solution for VM Migration and VM consolidation in a dynamic fashion. The dynamic consolidation was performed using historical data based on resource usage traces by VM to reduce energy and to ensure Service Level Agreement (SLA). 


\section{CLOUD COMPUTING DEPLOYMENT MODELS AND ITS SERVICES}

Cloud computing with its style where the resources will be provided as-a-service to multiple clients with elasticity and scalability using Internet technologies [15] present in figure 2. Cloud is a commercial model with technological capabilities where the user can consume compute, storage, network or application with metered consumption without nay capital investment in infrastructure. The cloud consumption has three different characteristics namely elastic and scalable, self-service and pay per use.

Cloud computing with utility computing can help the customers to plug in with components or applications because all the interfaces have been standardized between implementations.

\section{A. Deployment Models in Cloud}

\section{- Public Cloud}

It is an off-site location where the cloud resources are availed dynamically through web by means of web services or web applications based on request and demand [16]. The basic characteristics of public cloud are multi-tenancy which can be achieved using virtualization at various level of the software stack.

\section{- Private Cloud}

It is an on-premise cloud computing of an organization. Organization has a set-up of large data center within its premises and resources can be shared securely among the employees of that enterprise alone. Since public cloud may impose a security threat or breach for an organization and it can have accessed outside the enterprise boundary, private clouds are preferred. This type of cloud offers greater control over security.

\section{- Hybrid Cloud}

It is a combination of some properties of public cloud and some characteristics from private cloud i.e. some organization maintain their data confidentiality in private cloud and deploying or testing the application in public cloud. Hybrid cloud provides the opportunity and avail the resources that too expensive to maintain. An example is maintaining business application backups which are non-critical in public cloud for High Availability (HA). The hybrid cloud is ideal for security, flexibility and scalability.

\section{- Community Cloud}

Enterprises or organizations with same or mutual type of requirements use the community cloud infrastructure. They are specially built for some specific purpose for the compatible organizations to share the cloud environment to work on joint projects [17].

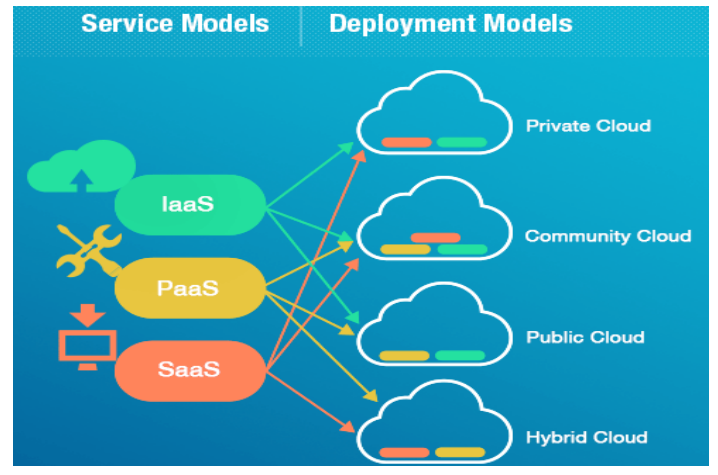

Figure 2: Cloud Deployment Models

\section{B. Service Models in Cloud and Its Benefits}

Cloud computing can render several services based on roles, user organization, and service providers. The service models in the cloud broadly classified based on the figure 3

\section{i. IAAS}

It is known as Infrastructure-as-a-Service. IaaS outsources the physical resources such as server, storage and network from third-party service provider. The resources are hosted on remote data center and the users can access through web API.

\section{Benefits:}

- More flexibility.

- Avoids Hardware maintenance on in-house.

- Resource Management is easy.

- Remote access is possible via Internet connection.

\section{ii. PAAS}

Platform-as-a-service allows outsourcing the software environment as well as physical hardware infrastructure which contains integration layers, databases, runtime and much more. This model is ideal for one who wants to have a control over business products and applications.

\section{Benefits:}

- Saves time on project developments.

- Quick and rapid production.

- Implementation of the platform can be managed automatically.

- Business data can be secured.

\section{iii.SAAS}

This Software-as-a-Service has been provided through Internet and no need for prior installation of software. This service can be obtained from any corner of the world at minimal cost. It is preferred by most of the companies since sales growth is high in cloud solutions.

\section{Benefits:}

- No installation or maintenance of software.

- Free from infrastructure maintenance and management, aligning with software updates.

- New software solutions enable easy and agile testing. 


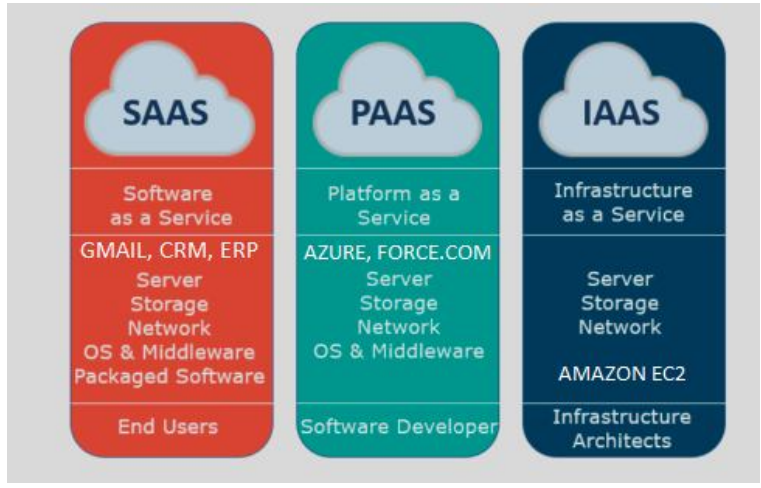

Figure 3: Services in Cloud Computing

\section{METHODLOGIES AND TECHNIQUES TURNING CLOUD INTO GREEN CLOUD}

Even though there are some great anxieties in the society that cloud computing consumes more energy usage in large data centers and produce more carbon emissions, cloud computing has some green tracing [18]. There are several techniques, metrics, technologies followed by cloud service providers to achieve greater efficiency and high utilization of resources to make the cloud green which has lower carbon emission rate because of modernizing the data centers into energy-efficient infrastructure by means of the below technologies discussed below figure 4:

\section{A. Multi-tenancy}

This approach overall reduces the energy usage and $\mathrm{CO}_{2}$ emissions in cloud computing infrastructure to make it green. The multiple organizations can share the same infrastructure and software by means of SaaS providers. This prevents redundancy of same software copies installed on various infrastructures. When there is a peak demand in business, multi-tenancy allows the same server to be shared to balance the variable demand patterns to eliminate the need of additional infrastructure. The exact prediction during fluctuation during demand leads to more energy savings.

\section{B. Dynamic provisioning}

On the demand basis the Cloud Service Providers (CSP) should predict with fast Fourier transformation and allocate the resources dynamically according to the requirement of applications. Sometimes an application may require fewer resources and at that time server consolidation can be done. To avoid over-provisioning Data Center (DC) always maintains active servers to meet the current demand. The dynamic provisioning can be done by allocating the resources from resource pool in the form of virtual machines. One of the dynamic provisioning mechanisms is template based architecture which orchestrates or automates the service provisioning using template description. It has three layers. The layer 1 has virtualized IT resources, layer 2 has service orchestrator for dynamic provisioning and layer 3 is an abstraction layer provides abstraction of interfaces (GUI) for managing various cloud providers and users with tools [19].

\section{Data center power efficiency}

The total power usage in data center has more impact on environment in cloud computing. So there is a need to design modern data center with advanced cooling systems and technologies to maintain Power Usage Efficiency (PUE) levels within the range from 1.1 to 1.2 which has $45 \%$ more efficiency than the siloed or complex traditional data center. The host design with virtual machines should support virtualization, container or bare metal for modern workloads with high power efficiency. The cooling system of data center should be in the form of water based system. The power management should be autonomic by means of intelligent control mechanism through software in Next Generation data centers.

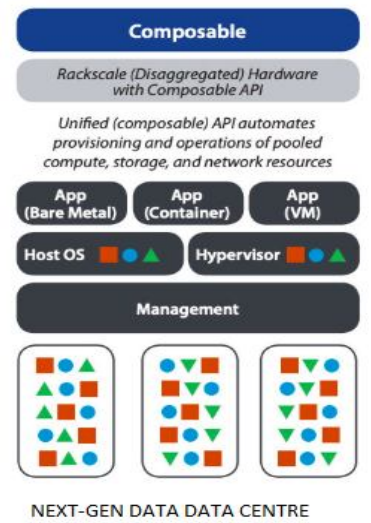

Figure 4: Next-Generation data center model

\section{Host Virtualization}

In on-premise infrastructure the server utilization is very low and the average utilization sometimes may reduce to 8 to 10 percent. On applying virtualization technologies on single host to run multiple applications to be executed with high level of utilization up to $65 \%$ greater and with more energy efficiency. Server virtualization considerably reduces the count of active physical servers.

\section{- Hypervisor role on virtualization}

Hypervisor is a piece of software which makes virtualization possible. It abstracts the guest machine and operating system (OS) from the existing hardware. Hypervisor is used to create a virtualization layer that isolates CPU, memory and other hardware resources form virtual machine which will be created. Host machine contains the hypervisor and guest machine that runs on the top of them. Virtualization is really a boon in cloud technology to turn as green cloud with significant less power usage and cost with sustainability.

- Types of Hypervisors

\section{i. Type-1 Hypervisor}

It is also known as native or bare-metal hypervisor. It runs directly on the top of the physical server and there is no software or operating system relies in between physical host and VM's is called bare-metal hypervisor. Type-1 hypervisor is particularly used in enterprise data centers for superior performance and stability with huge power savings. 


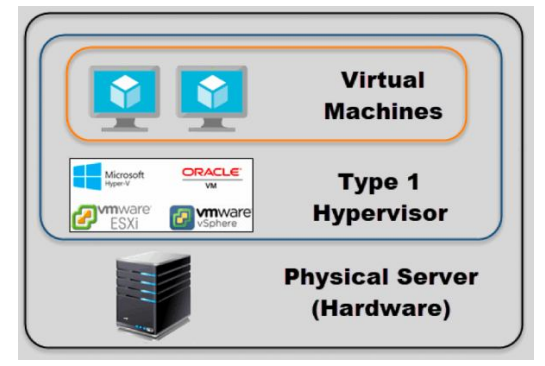

Figure 5: Type-1 Hypervisor

In figure 5, the most common Type-1 hypervisors are ESXi of VMware VSphere, KVM (Kernel-based Virtual Machine) built into Linux. This KVM has the functionalities such as scheduling, live migration, and resource control, Hyper-V of Microsoft, Citrix Hypervisor for enhanced virtualized graphics and with work security features.

\section{ii. Type-2 Hypervisor}

It runs inside the operating system of a physical server machine. It is also called hosted hypervisors. This kind of hypervisors is suitable for desktop virtualization for running parallel desktop and for small number of servers. Example for Type-2 hypervisor is Virtual Box by Oracle shown on figure 6.

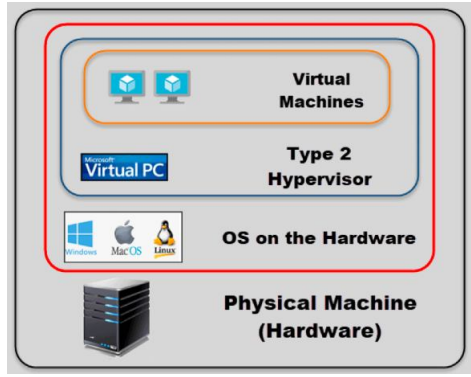

Figure 6: Type-2 Hypervisor

\section{E. Application container in $P O D$}

For the large benefit of energy efficiency container-as-a-service has deployed to make the cloud green. The Power Usage Efficiency (PUE) in the CISCO containerized data center also known as Performance Optimized Data center (POD) showed a gradual improvement from 1.05 to 1.25 [20]. Container startup time is very less on comparing the VM because application in container is partially separated from OS and container is application centric. Hence the containers are light-weight. The containerized data center delivers the self-contained DC anywhere virtually with reduced footprint. It supports live deployment. In figure 7, containers are gaining popularity for production in DC because it is an OS level virtualization. Containers also help the Dev-Ops teams in IT for rapid development of application which saves more cost and energy and to make the business agile. The containers can be orchestrated with kubernetes. Containers are scalable because it is well adapted to execute with micro-services. In POD cluster manager, container controller and workload scheduler should pool to get right capacity for modern workloads. Google Infrastructure running containers requires only less maintenance because only less number of OS versions is deployed across fleet of machines at a single time.

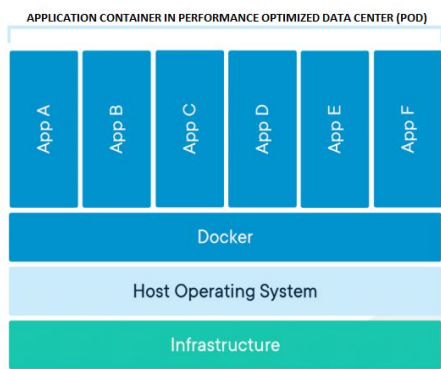

Figure 7: Container

\section{GREEN CLOUD ARCHITECTURE}

In this architecture, the user submits the request through a Green Broker which acts as a middleware to select greenest cloud service provider and also to service the user request. User may request for the services such as IaaS, SaaS or PaaS. The public directory consists of "Green offers" which can service the request registered by cloud service provider [24]. The public directory can also be accessed by green broker.

Green offers contain green services such as cost, time and when should be accessed for less carbon footprint. In this Green Cloud Architecture, carbon emission directory consists of energy metrics to calculate carbon emissions such as Power Usage Efficiency (PUE), Carbon Usage Effectiveness (CUE) etc. The green cloud framework is used to keep track of total energy usage to serve the user better. From the end-user part it is complex in selecting green cloud services with Quality of Service (QOS) rather each cloud layer should be "Green Aware" at the cloud service provider part shown in figure 8 .

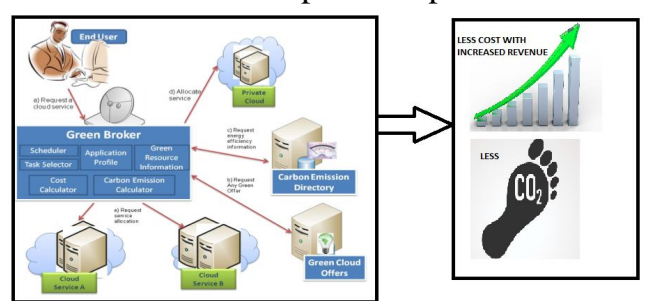

Figure 8: Green Cloud Architecture

\section{GREEN DATA CENTER}

Green data center should be designed and operated in such a way with air conditioning systems, IT systems, and electrical systems to support maximum energy efficiency and with low environmental impacts. The data center should use advanced cooling and It systems for operational and processing needs with energy efficiency. The data center energy consumption can be saved with the techniques of Green data storage, blade servers, advanced cooling concepts, Green networks and server clustering.

\section{A. Green Data Storage}

Storage requirements are drastically growing because of big data and the storage systems in the data center claims more amount of power and cooling cost. The storage system alone consumes 265 to $45 \%$ of total IT energy usage. For storing the persistent data with the improvement of energy efficiency by means of approaches such as Solid State Drives (SSD), tiered 
storage, Massive Amount of Independent Disk (MAID) and through Disk spinning Down (DSD). Data De-duplication technique helps to reduce the redundant data to improve storage utilization. It allows storing only the unique data. Data de-duplication performs and yield compression ratio within the range from 3:1 to 10:1. De-duplication helps to reduce the data by maintaining uniqueness and that must be sent across network for replication in various data centers for disaster recovery and for remote backups. Tiering technique is used to filter the unnecessary files and data. Tiering also helps to provide data immediately for corporate analytics. Tier-1 is used for on-demand data, tier-2 for timely and not critical data; tier-3 helps for archival data [23].

\section{B. Blade Server}

In some fundamental way blade systems are differed from rack systems. The rack system requires power and network cabling for each unit and they are self-contained stand-alone machines, whereas blade systems are kept within blade system container mounted in a standard cabinet vertically. The blade cabinet system contains on-board cooling and Uninterrupted Power Supply (UPS) provided by blade cabinet. Blade servers are very thin and it can be easily replaceable without disturbing others. PSSC labs announced its new type of eco-blade server which is most energy efficient server and can offer power savings up to $46 \%$ per server and also it minimizes physical space. Blade servers provide easy systems management via web browser [22].

\section{Green Network and communication}

The information sharing, online shopping, E- learning makes the communication networks on-demand which increases the energy consumption. The traditional network protocols are not designed specifically for energy conservation. It reflects negative impact on environment. Green Networking minimizes environmental impact by using eco-protocols, networking technologies and products. Some of the green networking practices are:

- Use brand new network products with energy compliance.

- Use smart systems and power management for energy efficient IT networks.

- Upgrade the device with energy-optimizing protocol design.

\section{Objectives of Green Networking}

- To reduce carbon footprint of wireless and delivery networks.

- Minimize the financial cost of operators to broadcast.

- Increasing operational sustainability and employing industry standards.

- Reduce the load on network to increase power efficiency.

Efficient Protocol objectives for network efficiency
Objective 1: Minimize the number of overhead packets corresponding with transmission.

Objective 2: Reduce the number of mandatory bits associated with per protocol.

Objective 3: Reduce the number of re-transmission attempts 'R'.

Objective 4: Increase the number of packet sent successfully from each node.

\section{Cooling Systems in Green Data Center}

Most IT servers and other equipment in data center produces heat. In the DC the aim of cooling ensures that over-heat of Information Technology (IT) equipment do not degrade or deface the performance which causes service interruptions. In data center, server rack faces cold aisle in front side and hot aisles in rear side.

The air-based system characterized by room, rack or row based. Rack-based systems are more efficient with power to supply flow of air confined to rack itself. On comparing room-based air cooling systems, row-based is more efficient. In Green Data Centers water-side or set-side cooling or chilling can be adopted for reduced energy consumption instead of mechanical refrigeration. In wet-side cooling the Heat Exchanger (HEX) returns the water alone and heat dissipates through dry cooler to external environment. Now-a-days the modern data centers are constructed with stern regulations near lake or river for waterside cooling to save more cost on cooling and to maintain sustainability on nature.

\section{E. Green Software sustainability}

It is an eco-friendly software to run in data centers to manage and monitor power management and it should be design with agile methodology, reusability of code and portability to run on different environments to reduce e-wastes and to enhance re-usability of modules without developing again from scratch which again saves time, cost and energy.

\section{GREEN CLOUD ENERGY METRICS}

The power usage in data centers ranges from few Kilowatts for a single rack and more megawatts for huge facilities. The power usage for resources in data center exceeds the actual power requirement. The metrics are always vital to increase operational efficiency (OPEX) to decrease economic cost.

\section{- PUE Metrics}

It is used to measure the data center power usage and efficiency consume by computing resources or equipment.

PUE $=$ Total Facility Power/ IT Component Power

\section{- Data Center Infrastructure Efficiency}

It is the reciprocal of PUE, i.e. DCiE=1/PUE=IT components power/Total Facility power*100. It is to be noted that Total Facility Power includes IT components, UPS, Chilling system, CPU, Storage and network nodes.

\section{Evolving Metrics for Green Data Center (DC) \\ - Carbon Usage Effectiveness (CUE)}


CUE estimates carbon emissions of a data center and factors to determine while design operation and location of data center. To calculate relative sustainability of a DC, Carbon usage effectiveness is combined with Power usage effectiveness. $\mathrm{CUE}=\mathrm{CO}_{2}$ released $\left(\mathrm{kgCO}_{2}\right.$ equivalent $) /$ unit of energy $(\mathrm{KWh}))^{*}$ (Sum of data center energy/IT resource energy)

\section{- Power Reuse Effectiveness (PRE)}

To recover waste energy and reuse it from data center while emitted during operations such as heat.

PRE $=($ Total Facility Power-Power Reuse $) /$ IT Resources energy.

\section{THIN CLIENT FOR GREEN CLOUD COMPUTING}

Thin client is offered as Desktop-as-a-service (DaaS) in cloud to attain "Go Green" approach. It is a Virtual Desktop Infrastructure (VDI) hosted by Cloud Service Provider (CSP). Thin clients always rely on host server in the data center to process the work through virtual desktop. Thin client consumes only less power on comparing Desktop PC's also known as fat clients because of more hardware kept inside the tower of PC. The cloud service providers deliver the operations for Virtual Desktop Interface (VDI). DaaS provider is responsible to maintain storage, OS upgrades and backup also to provide newer technology through pay-as-you-go model. In DaaS, remote display protocols offer high resolution sessions, dual monitor support, screen sharing, USB detection etc. Example: VM Horizon for Virtual Desktop Infrastructure.

Advantages of VDI:

\section{- Reduced Cost}

Lower in price with simple device.

- Lower Maintenance

Since thin client is having less components if anything goes wrong, it is easy to trouble shoot the problem.

- Easy Use and Access

User can select their own OS flavor according to their workload and expertisation and hence complexity is reduced in accessing.

- Security

Desktop solutions is administered and monitored by network administrators in data center $24 \times 7$ by centralized security management software.

\section{E-WASTE MANAGEMENT IN GREEN CLOUD COMPUTING}

From the figure 9, e-waste management in green cloud computing is the need of the hour that every individual particularly in IT and its other related domains, end-users to take a rigorous effort to follow 3 R's (Reduce, Reuse and Recycle) process [21].

Step 1: Reduce the amount of E-waste produced by means of implementing virtualization wherever possible.

Step 2: Reuse the discarded computer peripherals and electric parts after testing its fitness or make it ready to work again.

Step 3: Recycle the obsolete products with the direction of safe disposal mechanisms such as sorting components, categorizing for recycle or to reuse it, size-reduction to avoid dust emission from large parts, over-band magnet process for removing ionizing from steel, optical identification in plastic may contain brass or copper at their frames should be removed carefully.

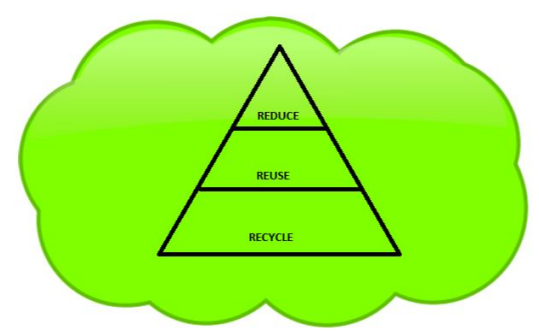

Figure 9: E-Waste Management by 3 R's

\section{CONCLUSION AND FUTURE WORK}

Even though cloud computing provides the users on-demand needs of resources as services through pay-as-you model, on the other side it leads to the alarming of Green House Gas (GHG) emissions. In this article it was discussed with various strategies, techniques, metrics to minimize the heat and $\mathrm{CO} 2$ and recycle mechanisms to attain the sustainable environment. It is not only enough to adopt these technologies for attaining Green Cloud Computing rather from the user perspective they should use the technologies and resources with right sense and through better power management options. The Cloud Service Provider (CSP) should take the massive efforts to modernize their data centers to cater the needs of the customers by supporting their traditional and modern workloads through providing the resources with agility to save cost and time. The data center should also be equipped with state-of-the-art facilities like using the natural resources like solar energy, river water for cooling etc. to balance the nature in eco-friendly way. By following these technologies with regulatory compliances in the data center an enterprise can save cost, energy and leads to more business agility. As a direction of future work, virtualization and containerization techniques will be implemented by designing an autonomic Software Defined Data Center (SDDC) to attain Green Cloud Computing.

\section{REFERENCES}

1. Garg, Saurabh Kumar, and Rajkumar Buyya. "Green cloud computing and environmental sustainability." 
Harnessing Green IT: Principles and Practices 2012 (2012): 315-340.

2. Buyya, Rajkumar, Chee Shin Yeo, and Srikumar Venugopal. "Market-oriented cloud computing: Vision, hype, and reality for delivering it services as computing utilities." In 2008 10th IEEE international conference on high performance computing and communications, pp. 5-13. Ieee, 2008.

3. Rivoire, Suzanne, Mehul A. Shah, Parthasarathy Ranganathan, and Christos Kozyrakis. "JouleSort: a balanced energy-efficiency benchmark." In Proceedings of the 2007 ACM SIGMOD international conference on Management of data, pp. 365-376. 2007.

4. Sami, Mostafa, M. Haggag, and Dina Salem. "Resource allocation and server consolidation algorithms for green computing." International Journal of Scientific \& Engineering Research 6, no. 12 (2015): 313-316.

5. Ganapathy, K., and M. N. Karuppusamy. "Cost Optimazation in Cloud Computing Based on Green Control Algorithm." IJETCSE, vol. 22, Issue 1, 2016.

6. Shakeel, Fatima, and Seema Sharma. "Green cloud computing: A review on efficiency of data centres and virtualization of servers." International Conference on Computing, Communication and Automation (ICCCA), pp. 1264-1267. IEEE, 2017..

7. Satoh, Fumiko, Hiroki Yanagisawa, Hitomi Takahashi, and Takayuki Kushida. "Total energy management system for cloud computing." In IEEE International Conference on Cloud Engineering (IC2E), pp. 233-240. IEEE, 2013.

8. Duy, Truong Vinh Truong, Yukinori Sato, and Yasushi Inoguchi. "Performance evaluation of a green scheduling algorithm for energy savings in cloud computing." In IEEE international symposium on parallel \& distributed processing, workshops and $\mathrm{Phd}$ forum (IPDPSW), pp. 1-8. IEEE, 2010.

9. Beik, Rasoul. "Green cloud computing: An energy-aware layer in software architecture." In Spring Congress on Engineering and Technology, pp. 1-4. IEEE, 2012.

10. Yamini, R. "Power management in cloud computing using green algorithm." In IEEE-International Conference On Advances In Engineering, Science And Management (ICAESM-2012), pp. 128-133. IEEE, 2012.

11. Nimje, Anup R., V. T. Gaikwad, and H. N. Datir. "Green Cloud Computing: A Virtualized Security Framework for Green Cloud Computing." International Journal of Advanced Research in Computer Science and Software Engineering 3, no. 4 (2013): 642-646.

12. Nimje, Anup R., V. T. Gaikwad, and H. N. Datir. "Green Cloud Computing: A Virtualized Security Framework for Green Cloud Computing." International Journal of Advanced Research in Computer Science and Software Engineering vol 3, no. 4 (2013): 642-646.
13. Anusuya, Krishnapriya, and V. K. Krishnapriya. "Green Cloud: A Pocket-Level Simulator with On-Demand Protocol for Energy-Aware Cloud Data Centers." International Journal of Science and Research (IJSR) 3, no. 2 (2014).

14. Anusuya, Krishnapriya, and V. K. Krishnapriya. "Green Cloud: A Pocket-Level Simulator with On-Demand Protocol for Energy-Aware Cloud Data Centers." International Journal of Science and Research (IJSR) 3, no. 2 (2014).

15. Plummer, Daryl C., David Mitchell Smith, Thomas J. Bittman, David W. Cearley, et al. "Five refining attributes of public and private cloud computing." Gartner Research 167182, no. 5 (2009).

16. Mell, Peter, and Timothy Grance. "Draft NIST working definition of cloud computing. National Institute of Standards and Technology." Information Technology Laboratory (2009).

17. Gouda, K., Anurag Patro, Dines Dwivedi, and Nagaraj Bhat. "Virtualization approaches in cloud computing." International Journal of Computer Trends and Technology (IJCTT) 12, no. 4 (2014): 161-166.

18. Murugesan, San. "Harnessing green IT: Principles and practices." IT professional 10, no. 1 (2008): 24-33. IEEE, ISBN: 978-81-265-3968-0, 2017.

19. Acharya, Shreenath, and Demian Antony D'Mello. "Cloud computing architectures and dynamic provisioning mechanisms." In 2013 International Conference on Green Computing, Communication and Conservation of Energy (ICGCE), pp. 798-804. IEEE, 2013.

20. The link, Containerized data centers solution overview available at: https://www.cisco.com/c/ dam/en_us/_soltuions/industries/docs/gov/ containerized_data_ centers_solution_overview.pdf, CISCO Solution, Overview, "Containerized Data centers: Compelling economics and efficiency".

21. Debnath, Biswajit, Reshma Roychoudhuri, and Sadhan K. Ghosh. "E-waste management-a potential route to green computing." Procedia Environmental Sciences 35 (2016): 669-675.

22. Kantilal P Rane, "Design of Drone3dContour: A Novel Contouring System using Altitude Measurement and Cloud-Web Computing”, International Journal of Emerging Trends in Engineering Research, Volume 8. No. 6, pp: 2395 - 2401, June 2020

23. Dominic Afuro Egbe, Bethel Mutanga Murimo, "A Resource-aware Service Discovery Architecture for Ad-hoc Mobile Cloud", International Journal of Emerging Trends in Engineering Research, Volume 8. No. 6, pp: 2402 - 2408, June 2020

24. K. Aruna Kumari, Dr.JKR Sastry, Dr. K Rajasekhara Rao, "An Enhanced Cloud Computing Architecture focused at Optimising VM Migration through an efficient Placement Algorithm", International Journal of Emerging Trends in Engineering Research, Volume 8. No. 6, pp: 2779- 2797, June 2020 